\title{
Multi-task Pairwise Neural Ranking for Hashtag Segmentation
}

\author{
Mounica Maddela ${ }^{1}$, Wei $\mathrm{Xu}^{1}$, Daniel Preoţiuc-Pietro ${ }^{2}$ \\ ${ }^{1}$ Department of Computer Science and Engineering, The Ohio State University \\ ${ }^{2}$ Bloomberg LP \\ \{maddela.4, xu.1265\}@osu.edu dpreotiucpie@bloomberg.net
}

\begin{abstract}
Hashtags are often employed on social media and beyond to add metadata to a textual utterance with the goal of increasing discoverability, aiding search, or providing additional semantics. However, the semantic content of hashtags is not straightforward to infer as these represent ad-hoc conventions which frequently include multiple words joined together and can include abbreviations and unorthodox spellings. We build a dataset of 12,594 hashtags split into individual segments and propose a set of approaches for hashtag segmentation by framing it as a pairwise ranking problem between candidate segmentations. ${ }^{1}$ Our novel neural approaches demonstrate $24.6 \%$ error reduction in hashtag segmentation accuracy compared to the current state-of-the-art method. Finally, we demonstrate that a deeper understanding of hashtag semantics obtained through segmentation is useful for downstream applications such as sentiment analysis, for which we achieved a $2.6 \%$ increase in average recall on the SemEval 2017 sentiment analysis dataset.
\end{abstract}

\section{Introduction}

A hashtag is a keyphrase represented as a sequence of alphanumeric characters plus underscore, preceded by the \# symbol. Hashtags play a central role in online communication by providing a tool to categorize the millions of posts generated daily on Twitter, Instagram, etc. They are useful in search, tracking content about a certain topic (Berardi et al., 2011; Ozdikis et al., 2012), or discovering emerging trends (Sampson et al., 2016).

Hashtags often carry very important information, such as emotion (Abdul-Mageed and Ungar,

\footnotetext{
${ }^{1}$ Our toolkit along with the code and data are publicly available at https://github.com/mounicam/ hashtag_master
}

\begin{tabular}{lcc}
\hline \multicolumn{1}{c}{ Type } & Single-token & Multi-token \\
\hline Named-entity (33.0\%) & \#lionhead & \#toyotaprius \\
\hline Events (14.8\%) & \#oscars & \#ipv6summit \\
\hline Standard (43.6\%) & \#snowfall & \#epicfall \\
\hline Non-standard (11.2\%) & \#sayin & \#iloveu4eva \\
\hline
\end{tabular}

Table 1: Examples of single- $(47.1 \%)$ and multi-word hashtags $(52.9 \%)$ and their categorizations based on a sample of our data.

2017), sentiment (Mohammad et al., 2013), sarcasm (Bamman and Smith, 2015), and named entities (Finin et al., 2010; Ritter et al., 2011). However, inferring the semantics of hashtags is nontrivial since many hashtags contain multiple tokens joined together, which frequently leads to multiple potential interpretations (e.g., lion head vs. lionhead). Table 1 shows several examples of single- and multi-token hashtags. While most hashtags represent a mix of standard tokens, named entities and event names are prevalent and pose challenges to both human and automatic comprehension, as these are more likely to be rare tokens. Hashtags also tend to be shorter to allow fast typing, to attract attention or to satisfy length limitations imposed by some social media platforms. Thus, they tend to contain a large number of abbreviations or non-standard spelling variations (e.g., \#iloveu4eva) (Han and Baldwin, 2011; Eisenstein, 2013), which hinders their understanding.

The goal of our study is to build efficient methods for automatically splitting a hashtag into a meaningful word sequence. Our contributions are: - A larger and better curated dataset for this task;

- Framing the problem as pairwise ranking using novel neural approaches, in contrast to previous work which ignored the relative order of candidate segmentations;

- A multi-task learning method that uses different sets of features to handle different types of 
hashtags;

- Experiments demonstrating that hashtag segmentation improves sentiment analysis on a benchmark dataset.

Our new dataset includes segmentation for 12,594 unique hashtags and their associated tweets annotated in a multi-step process for higher quality than the previous dataset of 1,108 hashtags (Bansal et al., 2015). We frame the segmentation task as a pairwise ranking problem, given a set of candidate segmentations. We build several neural architectures using this problem formulation which use corpus-based, linguistic and thesaurus based features. We further propose a multi-task learning approach which jointly learns segment ranking and single- vs. multi-token hashtag classification. The latter leads to an error reduction of $24.6 \%$ over the current state-of-the-art. Finally, we demonstrate the utility of our method by using hashtag segmentation in the downstream task of sentiment analysis. Feeding the automatically segmented hashtags to a state-of-the-art sentiment analysis method on the SemEval 2017 benchmark dataset results in a $2.6 \%$ increase in the official metric for the task.

\section{Background and Preliminaries}

Current approaches for hashtag segmentation can be broadly divided into three categories: (a) gazeteer and rule based (Maynard and Greenwood, 2014; Declerck and Lendvai, 2015; Billal et al., 2016), (b) word boundary detection (Çelebi and Özgür, 2017, 2016), and (c) ranking with language model and other features (Wang et al., 2011; Bansal et al., 2015; Berardi et al., 2011; Reuter et al., 2016; Simeon et al., 2016). Hashtag segmentation approaches draw upon work on compound splitting for languages such as German or Finnish (Koehn and Knight, 2003) and word segmentation (Peng and Schuurmans, 2001) for languages with no spaces between words such as Chinese (Sproat and Shih, 1990; Xue and Shen, 2003). Similar to our work, Bansal et al. (2015) extract an initial set of candidate segmentations using a sliding window, then rerank them using a linear regression model trained on lexical, bigram and other corpus-based features. The current state-ofthe-art approach (Çelebi and Özgür, 2017, 2016) uses maximum entropy and CRF models with a combination of language model and hand-crafted features to predict if each character in the hashtag is the beginning of a new word.

Generating Candidate Segmentations. Microsoft Word Breaker (Wang et al., 2011) is, among the existing methods, a strong baseline for hashtag segmentation, as reported in Çelebi and Özgür (2017) and Bansal et al. (2015). It employs a beam search algorithm to extract $k$ best segmentations as ranked by the n-gram language model probability:

$$
\operatorname{Score}^{L M}(s)=\sum_{i=1}^{n} \log P\left(w_{i} \mid w_{i-N+1} \ldots w_{i-1}\right)
$$

where $\left[w_{1}, w_{2} \ldots w_{n}\right]$ is the word sequence of segmentation $s$ and $N$ is the window size. More sophisticated ranking strategies, such as Binomial and word length distribution based ranking, did not lead to a further improvement in performance (Wang et al., 2011). The original Word Breaker was designed for segmenting URLs using language models trained on web data. In this paper, we reimplemented ${ }^{2}$ and tailored this approach to segmenting hashtags by using a language model specifically trained on Twitter data (implementation details in §3.6). The performance of this method itself is competitive with state-of-the-art methods (evaluation results in $\S 5.3$ ). Our proposed pairwise ranking method will effectively take the top $k$ segmentations generated by this baseline as candidates for reranking.

However, in prior work, the ranking scores of each segmentation were calculated independently, ignoring the relative order among the top $k$ candidate segmentations. To address this limitation, we utilize a pairwise ranking strategy for the first time for this task and propose neural architectures to model this.

\section{Multi-task Pairwise Neural Ranking}

We propose a multi-task pairwise neural ranking approach to better incorporate and distinguish the relative order between the candidate segmentations of a given hashtag. Our model adapts to address single- and multi-token hashtags differently via a multi-task learning strategy without requiring additional annotations. In this section, we describe the task setup and three variants of pairwise neural ranking models (Figure 1).

\footnotetext{
${ }^{2}$ To the best of our knowledge, Microsoft discontinued its Word Breaker and Web Ngram API services in early 2018.
} 


\begin{tabular}{r}
\hline hashtag $(h) \quad$ \#songsonghaddafisitunes \\
\hline $\begin{array}{r}\text { segmentation }\left(s^{*}\right) \\
(\text { i.e. songs on Ghaddafi s itunes }\end{array}$ \\
\hline candidate segmentations $(s \in S)$ \\
songs on ghaddafis itunes \\
songs on ghaddafisi tunes \\
songs on ghaddaf is itunes \\
song song haddafis i tunes \\
songsong haddafisitunes \\
(and ...)
\end{tabular}

Table 2: Example hashtag along with its gold and possible candidate segmentations.

\subsection{Segmentation as Pairwise Ranking}

The goal of hashtag segmentation is to divide a given hashtag $h$ into a sequence of meaningful words $s^{*}=\left[w_{1}, w_{2}, \ldots, w_{n}\right]$. For a hashtag of $r$ characters, there are a total of $2^{r-1}$ possible segmentations but only one, or occasionally two, of them $\left(s^{*}\right)$ are considered correct (Table 2).

We transform this task into a pairwise ranking problem: given $k$ candidate segmentations $\left\{s_{1}, s_{2}, \ldots, s_{k}\right\}$, we rank them by comparing each with the rest in a pairwise manner. More specifically, we train a model to predict a real number $g\left(s_{a}, s_{b}\right)$ for any two candidate segmentations $s_{a}$ and $s_{b}$ of hashtag $h$, which indicates $s_{a}$ is a better segmentation than $s_{b}$ if positive, and vice versa. To quantify the quality of a segmentation in training, we define a gold scoring function $g^{*}$ based on the similarities with the ground-truth segmentation $s^{*}$ :

$$
g^{*}\left(s_{a}, s_{b}\right)=\operatorname{sim}\left(s_{a}, s^{*}\right)-\operatorname{sim}\left(s_{b}, s^{*}\right) .
$$

We use the Levenshtein distance (minimum number of single-character edits) in this paper, although it is possible to use other similarity measurements as alternatives. We use the top $k$ segmentations generated by Microsoft Word Breaker $(\S 2)$ as initial candidates.

\subsection{Pairwise Neural Ranking Model}

For an input candidate segmentation pair $\left\langle s_{a}, s_{b}\right\rangle$, we concatenate their feature vectors $\mathbf{s}_{a}$ and $\mathbf{s}_{b}$, and feed them into a feedforward network which emits a comparison score $g\left(s_{a}, s_{b}\right)$. The feature vector $\mathbf{s}_{a}$ or $\mathbf{s}_{b}$ consists of language model probabilities using Good-Turing (Good, 1953) and modified Kneser-Ney smoothing (Kneser and Ney, 1995; Chen and Goodman, 1999), lexical and linguistic features (more details in $\$ 3.5$ ). For training, we use all the possible pairs $\left\langle s_{a}, s_{b}\right\rangle$ of the $k$ candidates as the input and their gold scores $g^{*}\left(s_{a}, s_{b}\right)$ as the target. The training objective is to minimize the Mean Squared Error (MSE):

$$
L_{M S E}=\frac{1}{m} \sum_{i=1}^{m}\left(g^{*(i)}\left(s_{a}, s_{b}\right)-\hat{g}^{(i)}\left(s_{a}, s_{b}\right)\right)^{2}
$$

where $m$ is the number of training examples.

To aggregate the pairwise comparisons, we follow a greedy algorithm proposed by Cohen et al. (1998) and used for preference ranking (Parakhin and Haluptzok, 2009). For each segmentation $s$ in the candidate set $S=\left\{s_{1}, s_{2}, \ldots, s_{k}\right\}$, we calculate a single score $\operatorname{Score}^{P N R}(s)=$ $\sum_{s \neq s_{j} \in S} g\left(s, s_{j}\right)$, and find the segmentation $s_{\max }$ corresponding to the highest score. We repeat the same procedure after removing $s_{\max }$ from $S$, and continue until $S$ reduces to an empty set. Figure 1(a) shows the architecture of this model.

\subsection{Margin Ranking (MR) Loss}

As an alternative to the pairwise ranker ( $(3.2)$, we propose a pairwise model which learns from candidate pairs $\left\langle s_{a}, s_{b}\right\rangle$ but ranks each individual candidate directly rather than relatively. We define a new scoring function $g^{\prime}$ which assigns a higher score to the better candidate, i.e., $g^{\prime}\left(s_{a}\right)>g^{\prime}\left(s_{b}\right)$, if $s_{a}$ is a better candidate than $s_{b}$ and vice-versa. Instead of concatenating the features vectors $\mathbf{s}_{a}$ and $\mathbf{s}_{b}$, we feed them separately into two identical feedforward networks with shared parameters. During testing, we use only one of the networks to rank the candidates based on the $g^{\prime}$ scores. For training, we add a ranking layer on top of the networks to measure the violations in the ranking order and minimize the Margin Ranking Loss (MR):

$$
\begin{aligned}
L_{M R} & =\frac{1}{m} \sum_{i=1}^{m} \max \left(0,1-l_{a b}^{(i)} p_{a b}^{(i)}\right) \\
p_{a b}^{(i)} & =\left(\hat{g}^{\prime(i)}\left(s_{a}\right)-\hat{g}^{\prime(i)}\left(s_{b}\right)\right) \\
l_{a b} & = \begin{cases}1 & g^{*}\left(s_{a}, s_{b}\right)>0 \\
-1 & g^{*}\left(s_{a}, s_{b}\right)<0 \\
0 & \text { otherwise }\end{cases}
\end{aligned}
$$

where $m$ is the number of training samples. The architecture of this model is presented in Figure 1(b).

\subsection{Adaptive Multi-task Learning}

Both models in $\S 3.2$ and $\S 3.3$ treat all the hashtags uniformly. However, different features address different types of hashtags. By design, the linguistic features capture named entities and multiword hashtags that exhibit word shape patterns, 


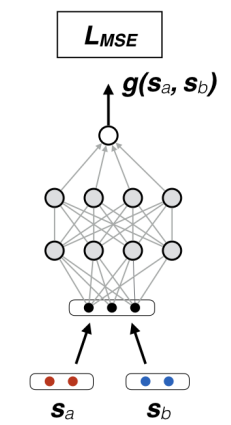

(a) Pairwise Ranking Model (MSE $§ 3.2$ )

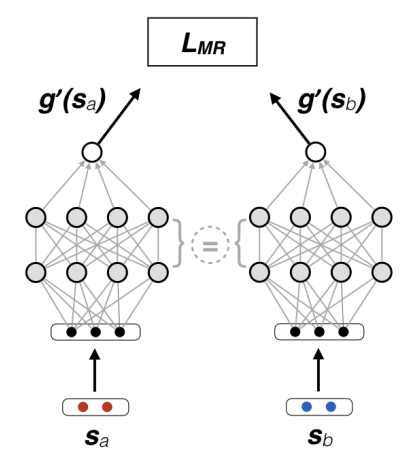

(b) Margin Ranking Loss w/ shared parameters (MR $\S 3.3)$

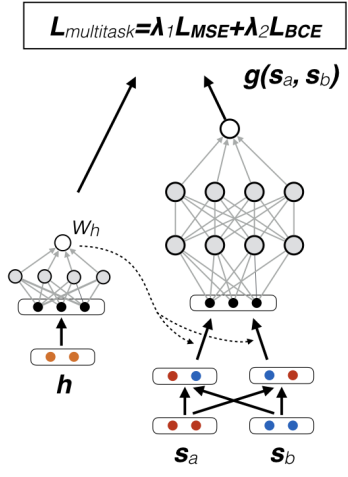

(c) Adaptive Multi-task Learning for Pairwise ranking (MSE+Multitask $\S 3.4$ )

Figure 1: Pairwise neural ranking models for hashtag segmentation. Given two candidate segmentations $s_{a}$ and $s_{b}$ of hashtag $h$, the goal is to predict the segmentation's goodness relative score $(g)$ or absolute $\left(g^{\prime}\right)$ score.

such as camel case. The ngram probabilities with Good-Turing smoothing gravitate towards multiword segmentations with known words, as its estimate for unseen ngrams depends on the fraction of ngrams seen once which can be very low (Heafield, 2013). The modified Kneser-Ney smoothing is more likely to favor segmentations that contain rare words, and single-word segmentations in particular. Please refer to $\S 5.3$ for a more detailed quantitative and qualitative analysis.

To leverage this intuition, we introduce a binary classification task to help the model differentiate single-word from multi-word hashtags. The binary classifier takes hashtag features $\mathbf{h}$ as the input and outputs $w_{h}$, which represents the probability of $h$ being a multi-word hashtag. $w_{h}$ is used as an adaptive gating value in our multitask learning setup. The gold labels for this task are obtained at no extra cost by simply verifying whether the ground-truth segmentation has multiple words. We train the pairwise segmentation ranker and the binary single- vs. multi-token hashtag classifier jointly, by minimizing $L_{M S E}$ for the pairwise ranker and the Binary Cross Entropy Error $\left(L_{B C E}\right)$ for the classifier:

$$
\begin{aligned}
& L_{\text {multitask }}=\lambda_{1} L_{M S E}+\lambda_{2} L_{B C E} \\
& L_{B C E}=-\frac{1}{m} \sum_{i=1}^{m}\left[l^{(i)} * \log \left(w_{h}^{(i)}\right)+\right. \\
&\left.\left(1-l^{(i)}\right) * \log \left(1-w_{h}^{(i)}\right)\right]
\end{aligned}
$$

where $w_{h}$ is the adaptive gating value, $l \in\{0,1\}$ indicates if $h$ is actually a multi-word hashtag and $m$ is the number of training examples. $\lambda_{1}$ and $\lambda_{2}$ are the weights for each loss. For our experiments, we apply equal weights.
More specifically, we divide the segmentation feature vector $\mathbf{s}_{a}$ into two subsets: (a) $\mathbf{s}_{a}^{K N}$ with modified Kneser-Ney smoothing features, and (b) $\mathbf{s}_{a}^{G L}$ with Good-Turing smoothing and linguistic features. For an input candidate segmentation pair $\left\langle s_{a}, s_{b}\right\rangle$, we construct two pairwise vectors $\mathbf{s}_{a b}^{K N}=$ $\left[\mathbf{s}_{a}^{K N} ; \mathbf{s}_{b}^{K N}\right]$ and $\mathbf{s}_{a b}^{G L}=\left[\mathbf{s}_{a}^{G L} ; \mathbf{s}_{b}^{G L}\right]$ by concatenation, then combine them based on the adaptive gating value $w_{h}$ before feeding them into the feedforward network $G$ for pairwise ranking:

$$
\hat{g}\left(s_{a}, s_{b}\right)=G\left(w_{h} \mathbf{s}_{a b}^{G L}+\left(1-w_{h}\right) \mathbf{s}_{a b}^{K N}\right)
$$

We use summation with padding, as we find this simple ensemble method achieves similar performance in our experiments as the more complex multi-column networks (Ciresan et al., 2012). Figure 1(c) shows the architecture of this model. An analogue multi-task formulation can also be used for the Margin Ranking loss as:

$$
L_{\text {multitask }}=\lambda_{1} L_{M R}+\lambda_{2} L_{B C E} .
$$

\subsection{Features}

We use a combination of corpus-based and linguistic features to rank the segmentations. For a candidate segmentation $s$, its feature vector $\mathbf{s}$ includes the number of words in the candidate, the length of each word, the proportion of words in an English dictionary ${ }^{3}$ or Urban Dictionary ${ }^{4}$ (Nguyen et al., 2018), ngram counts from Google Web 1TB corpus (Brants and Franz, 2006), and ngram probabilities from trigram language models trained on the Gigaword corpus (Graff and Cieri, 2003) and

\footnotetext{
${ }^{3}$ https: / /pypi.org/project/pyenchant

${ }^{4}$ https : / / www . urbandictionary. com
} 
1.1 billion English tweets from 2010, respectively. We train two language models on each corpus: one with Good-Turing smoothing using SRILM (Stolcke, 2002) and the other with modified KneserNey smoothing using KenLM (Heafield, 2011). We also add boolean features, such as if the candidate is a named-entity present in the list of Wikipedia titles, and if the candidate segmentation $s$ and its corresponding hashtag $h$ satisfy certain word-shapes (more details in appendix A.1).

Similarly, for hashtag $h$, we extract the feature vector $h$ consisting of hashtag length, ngram count of the hashtag in Google 1TB corpus (Brants and Franz, 2006), and boolean features indicating if the hashtag is in an English dictionary or Urban Dictionary, is a named-entity, is in camel case, ends with a number, and has all the letters as consonants. We also include features of the bestranked candidate by the Word Breaker model.

\subsection{Implementation Details}

We use the PyTorch framework to implement our multi-task pairwise ranking model. The pairwise ranker consists of an input layer, three hidden layers with eight nodes in each layer and hyperbolic tangent (tanh) activation, and a single linear output node. The auxiliary classifier consists of an input layer, one hidden layer with eight nodes and one output node with sigmoid activation. We use the Adam algorithm (Kingma and Ba, 2014) for optimization and apply a dropout of 0.5 to prevent overfitting. We set the learning rate to 0.01 and 0.05 for the pairwise ranker and auxiliary classifier respectively. For each experiment, we report results obtained after 100 epochs.

For the baseline model used to extract the $k$ initial candidates, we reimplementated the Word Breaker (Wang et al., 2011) as described in $\S 2$ and adapted it to use a language model trained on 1.1 billion tweets with Good-Turing smoothing using SRILM (Stolcke, 2002) to give a better performance in segmenting hashtags ( $\$ 5.3)$. For all our experiments, we set $k=10$.

\section{Hashtag Segmentation Data}

We use two datasets for experiments (Table 3): (a) STAN $N_{\text {small }}$, created by Bansal et al. (2015), which consists of 1,108 unique English hashtags from 1,268 randomly selected tweets in the Stanford Sentiment Analysis Dataset (Go and Huang, 2009) along with their crowdsourced segmentations and

\begin{tabular}{rcrcc}
\hline Data & $\begin{array}{r}\text { num. of Hashtags } \\
\text { (multi-token\%) }\end{array}$ & $\begin{array}{c}\text { avg. } \\
\text { \#char }\end{array}$ & $\begin{array}{c}\text { avg. } \\
\text { \#word }\end{array}$ \\
\hline \multirow{3}{*}{$S T A N_{\text {large }}$} & Train & $2518(51.9 \%)$ & 8.5 & 1.8 \\
& Dev & $629(52.3 \%)$ & 8.4 & 1.7 \\
& Test & $9447(53.0 \%)$ & 8.6 & 1.8 \\
\hline$S T A N_{\text {small }}$ & Test & $1108(60.5 \%)$ & 9.0 & 1.9 \\
\hline
\end{tabular}

Table 3: Statistics of the $S T A N_{\text {small }}$ and STAN large datasets - number of unique hashtags, percentage of multi-token hashtags, average length of hashtags in characters and words.

our additional corrections; and (b) STAN $N_{\text {large }}$, our new expert curated dataset, which includes all 12,594 unique English hashtags and their associated tweets from the same Stanford dataset.

Dataset Analysis. STAN $N_{\text {small }}$ is the most commonly used dataset in previous work. However, after reexamination, we found annotation errors in $6.8 \%{ }^{5}$ of the hashtags in this dataset, which is significant given that the error rate of the state-of-theart models is only around $10 \%$. Most of the errors were related to named entities. For example, \#lionhead, which refers to the "Lionhead" video game company, was labeled as "lion head".

Our Dataset. We therefore constructed the STAN $_{\text {large }}$ dataset of 12,594 hashtags with additional quality control for human annotations. We displayed a tweet with one highlighted hashtag on the Figure-Eight ${ }^{6}$ (previously known as CrowdFlower) crowdsourcing platform and asked two workers to list all the possible segmentations. For quality control on the platform, we displayed a test hashtag in every page along with the other hashtags. If any annotator missed more than $20 \%$ of the test hashtags, then they were not allowed to continue work on the task. For $93.1 \%$ of the hashtags, the workers agreed on the same segmentation. We further asked three in-house annotators (not authors) to cross-check the crowdsourced annotations using a two-step procedure: first, verify if the hashtag is a named entity based on the context of the tweet; then search on Google to find the correct segmentation(s). We also asked the same annotators to fix the errors in STAN $N_{\text {small }}$. The human upperbound of the task is estimated at $\sim 98 \%$ accuracy, where we consider the crowdsourced segmentations (two workers merged) as correct if at least one of them matches with our expert annotator's segmentations.

\footnotetext{
${ }^{5}$ More specifically, $4.8 \%$ hashtags is missing one of the two acceptable segmentations and another $2.0 \%$ is incorrect segmentation.

${ }^{6}$ https://figure-eight.com
} 


\begin{tabular}{|c|c|c|c|c|c|c|c|c|c|c|c|}
\hline & \multicolumn{4}{|c|}{ All Hashtags } & \multicolumn{4}{|c|}{ Multi-token } & \multicolumn{3}{|c|}{ Single-token } \\
\hline & $\mathbf{A} @ \mathbf{1}$ & $\mathbf{F}_{1} @ \mathbf{1}$ & A@2 & MRR & $\mathbf{A} @ \mathbf{1}$ & $\mathbf{F}_{1} @ \mathbf{1}$ & $\mathbf{A @ 2}$ & MRR & $\mathbf{A @ 1}$ & $\mathbf{A} @ 2$ & MRR \\
\hline Original hashtag & 51.0 & 51.0 & - & - & 19.1 & 19.1 & - & - & 100.0 & - & - \\
\hline Rule-based (Billal et a & 58.1 & 63.5 & - & - & 57.6 & 66.5 & - & - & 58.8 & - & - \\
\hline GATH & 73.2 & 77.2 & - & - & 71.4 & 78.0 & - & - & 76.0 & - & - \\
\hline Viter & 73.4 & 78.5 & - & - & 74.5 & 83.1 & - & - & 71.6 & - & - \\
\hline MaxF & 92.4 & 93.4 & - & - & 91.9 & 93.6 & - & - & 93.1 & - & - \\
\hline Wor & 90.8 & 91.7 & 97.4 & 94.5 & 88.5 & 90.0 & 97.8 & 93.7 & 94.3 & 96.8 & 95.7 \\
\hline Pair & 88.1 & 89.9 & 97.2 & 93.1 & 83.8 & 86.8 & 97.3 & 91. & 94.7 & 97.0 & 95.9 \\
\hline Pair & 92.3 & 93.5 & 98.2 & 95.4 & 90.9 & 92.8 & 99.0 & 95. & 94.5 & 96.9 & 95.8 \\
\hline Pairy & 92.5 & 93.7 & 98.2 & 95.5 & 91.2 & 93.1 & 99.0 & 95.4 & 94.5 & 97.0 & 95.8 \\
\hline Pairy & 93.0 & 94.3 & 97.8 & 95.7 & 91.5 & 93.7 & 98.7 & 95.4 & 95.2 & 96.6 & 96.0 \\
\hline Pairw & 94.5 & 95.2 & 98.4 & 96.6 & 93.9 & 95.1 & 99.4 & 96.8 & 95.4 & 96.8 & 96.2 \\
\hline Human Upperbound & 98.0 & 98.3 & - & - & 97.8 & 98.2 & - & - & 98.4 & - & - \\
\hline
\end{tabular}

Table 4: Evaluation results on the corrected version of $S T A N_{\text {small }}$. For reference, on the original version of $S T A N_{\text {small }}$, the Microsoft Word Breaker API reported an $84.6 \% \mathrm{~F}_{1}$ score and an $83.6 \%$ accuracy for the top one output (Çelebi and Özgür, 2017), while our best model (MSE+multitask) reported $89.8 \% \mathrm{~F}_{1}$ and $91.0 \%$ accuracy.

\begin{tabular}{|c|c|c|c|c|c|c|c|c|c|c|c|}
\hline & \multicolumn{4}{|c|}{ All Hashtags } & \multicolumn{4}{|c|}{ Multi-token } & \multicolumn{3}{|c|}{ Single-token } \\
\hline & $\mathbf{A @ 1}$ & $\overline{\mathbf{F}_{1} @ \mathbf{1}}$ & $\mathbf{A @ 2}$ & MRR & $\overline{\mathbf{A} @ 1}$ & $\mathbf{F} \mathbf{F}_{1} @ \mathbf{1}$ & $\overline{\mathbf{A} @ \mathbf{2}}$ & MRR & $\overline{\mathbf{A} @ 1}$ & $\mathbf{A @ 2}$ & MRR \\
\hline Original hashtag & 55.5 & 55.5 & - & - & 16.2 & 16.2 & - & - & 100.0 & - & - \\
\hline Rule-based (Billal et al., 2016) & 56.1 & 61.5 & - & - & 56.0 & 65.8 & - & - & 56.3 & - & - \\
\hline Viterbi (Berardi et a & 68.4 & 73.8 & - & - & 71.2 & 81.5 & - & - & 65.0 & - & - \\
\hline GATE Hashtą & 72.4 & 76.1 & - & - & 70.0 & 76.8 & - & - & 75.3 & - & - \\
\hline MaxEnt (Çelebi and Özgür, 20 & 91.2 & 92.3 & - & - & 90.2 & 92.4 & - & - & 92.3 & - & - \\
\hline Word Breaker & 90.1 & 91.0 & 96.6 & 93. & 88.5 & 90.0 & 97.0 & 93.4 & 91.9 & 96.2 & 94.4 \\
\hline Pairy & 89.2 & 91.1 & 96.3 & 93.3 & 84.2 & 87.8 & 95.6 & 91. & 94.8 & 97.0 & 95.9 \\
\hline Pairy & 91.3 & 92.6 & 97.2 & 94.6 & 89.9 & 92.4 & 97.5 & 94.3 & 92.8 & 96.8 & 94.9 \\
\hline Pairy & 91.3 & 92.6 & 97.0 & 94.5 & 91.0 & 93.6 & 97.7 & 94.9 & 91.5 & 96.2 & 94.1 \\
\hline Pairv & 91.4 & 92.7 & 97.2 & 94.6 & 90.0 & 92.6 & 97.7 & 94.4 & 92.9 & 96.6 & 94.9 \\
\hline Pairwise neural ranker (MSE+multitask) & 92.4 & 93.6 & 97.3 & 95.2 & 91.9 & 94.1 & 98.0 & 95.4 & 93.0 & 96.5 & 94.9 \\
\hline Human Upperbound & 98.6 & 98.8 & - & - & 98.0 & 98.4 & - & - & 99.2 & - & - \\
\hline
\end{tabular}

Table 5: Evaluation results on our $S T A N_{\text {large }}$ test dataset. For single-token hashtags, the token-level $\mathrm{F}_{1} @ 1$ is equivalent to segmentation-level A@1. For multi-token cases, A@1 and $\mathrm{F}_{1} @ 1$ for the original hashtag baseline are non-zero because $11.4 \%$ of the hashtags have more than one acceptable segmentations. Our best model (MSE+multitask) shows a statistically significant improvement $(p<0.05)$ over the state-of-the-art approach (Çelebi and Özgür, 2017) based on the paired bootstrap test (Berg-Kirkpatrick et al., 2012).

\section{Experiments}

In this section, we present experimental results that compare our proposed method with the other state-of-the-art approaches on hashtag segmentation datasets. The next section will show experiments of applying hashtag segmentation to the popular task of sentiment analysis.

\subsection{Existing Methods}

We compare our pairwise neural ranker with the following baseline and state-of-the-art approaches:

(a) The original hashtag as a single token;

(b) A rule-based segmenter, which employs a set of word-shape rules with an English dictionary (Billal et al., 2016);

(c) A Viterbi model which uses word frequencies from a book corpus ${ }^{7}$ (Berardi et al., 2011);

\footnotetext{
${ }^{7}$ Project Gutenberg http://norvig.com/big.txt
}

(d) The specially developed GATE Hashtag Tokenizer from the open source toolkit, ${ }^{8}$ which combines dictionaries and gazetteers in a Viterbi-like algorithm (Maynard and Greenwood, 2014);

(e) A maximum entropy classifier (MaxEnt) trained on the $S T A N_{\text {large }}$ training dataset. It predicts whether a space should be inserted at each position in the hashtag and is the current state-of-the-art (Çelebi and Özgür, 2017);

(f) Our reimplementation of the Word Breaker algorithm which uses beam search and a Twitter ngram language model (Wang et al., 2011);

(g) A pairwise linear ranker which we implemented for comparison purposes with the same features as our neural model, but using perceptron as the underlying classifier (Hopkins and May, 2011) and minimizing the hinge

\footnotetext{
${ }^{8}$ https://gate.ac.uk/
} 


\begin{tabular}{lcccccccc}
\hline & \multicolumn{2}{c}{ Single } & & \multicolumn{2}{c}{ Multi } & & \multicolumn{2}{c}{ All } \\
\cline { 2 - 3 } & $\mathbf{A}$ & MRR & & A & MRR & & A & MRR \\
\hline Kneser-Ney & $\mathbf{9 5 . 4}$ & $\mathbf{9 5 . 7}$ & & 56.0 & 75.3 & & 74.9 & 85.1 \\
Good-Turing (GT) & 91.4 & 93.5 & & 85.9 & 91.8 & & 88.6 & 92.6 \\
Linguistic (Ling) & 89.4 & 91.7 & & 71.6 & 82.6 & & 80.1 & 87.0 \\
GT + Ling & 92.4 & 93.9 & & $\mathbf{8 6 . 2}$ & $\mathbf{9 2 . 3}$ & & $\mathbf{8 8 . 9}$ & $\mathbf{9 2 . 7}$ \\
\hline All Features & 91.1 & 93.1 & & 89.0 & 93.7 & & 90.0 & 93.4 \\
\hline
\end{tabular}

Table 6: Evaluation of automatic hashtag segmentation (MSE) with different features on the STAN large dev set. A denotes accuracy@1. While Kneser-Ney features perform well on single-token hashtags, GT+Ling features perform better on multi-token hashtags.

loss between $g^{*}$ and a scoring function similar to $g^{\prime}$. It is trained on the $S T A N_{\text {large }}$ dataset.

\subsection{Evaluation Metrics}

We evaluate the performance by the top $k$ ( $k=$ 1,2) accuracy (A@1, A@2), average token-level $\mathrm{F}_{1}$ score $\left(\mathbf{F}_{1} @ \mathbf{1}\right)$, and mean reciprocal rank (MRR). In particular, the accuracy and MRR are calculated at the segmentation-level, which means that an output segmentation is considered correct if and only if it fully matches the human segmentation. Average token-level $\mathrm{F}_{1}$ score accounts for partially correct segmentation in the multi-token hashtag cases.

\subsection{Results}

Tables 4 and 5 show the results on the $S T A N_{\text {small }}$ and $S T A N_{\text {large }}$ datasets, respectively. All of our pairwise neural rankers are trained on the 2,518 manually segmented hashtags in the training set of $S T A N_{\text {large }}$ and perform favorably against other state-of-the-art approaches. Our best model (MSE+multitask) that utilizes different features adaptively via a multi-task learning procedure is shown to perform better than simply combining all the features together (MR and MSE). We highlight the $24.6 \%$ error reduction on $S T A N_{\text {small }}$ and $16.5 \%$ on $S T A N_{\text {large }}$ of our approach over the previous SOTA (Çelebi and Özgür, 2017) on the Multi-token hashtags, and the importance of having a separate evaluation of multi-word cases as it is trivial to obtain $100 \%$ accuracy for Singletoken hashtags. While our hashtag segmentation model is achieving a very high accuracy@2, to be practically useful, it remains a challenge to get the top one predication exactly correct. Some hashtags are very difficult to interpret, e.g., \#BTVSMB refers to the Social Media Breakfast (SMB) in Burlington, Vermont (BTV).

The improved Word Breaker with our addition of a Twitter-specific language model is a very strong

\begin{tabular}{lllll} 
& & & & \\
\hline
\end{tabular}

Table 7: Error (०) and correct $(\bullet)$ segmentation analysis of three pairwise ranking models (MSE) trained with different feature sets Each row corresponds to one area in the Venn diagram; for example, ০০০ is the set of hashtags that all three models failed in the STAN $N_{\text {large }}$ dev data and $\bullet \circ \circ$ is the set of hashtags that only the model with Kneser-Ney language model features (but not the other two models) segmented correctly.

baseline, which echos the findings of the original Word Breaker paper (Wang et al., 2011) that having a large in-domain language model is extremely helpful for word segmentation tasks. It is worth noting that the other state-of-the-art system (Çelebi and Özgür, 2017) also utilized a 4-gram language model trained on 476 million tweets from 2009.

\subsection{Analysis and Discussion}

Feature Analysis. To empirically illustrate the effectiveness of different features on different types of hashtags, we show the results for models using individual feature sets in pairwise ranking models (MSE) in Table 6. Language models with modified Kneser-Ney smoothing perform best on single-token hashtags, while Good-Turing and Linguistic features work best on multi-token hashtags, confirming our intuition about their usefulness in a multi-task learning approach. Table 7 shows a qualitative analysis with the first column (০o০) indicating which features lead to correct or wrong segmentations, their count in our data and illustrative examples with human segmentation.

Length of Hashtags. As expected, longer hashtags with more than three tokens pose greater challenges and the segmentation-level accuracy of our best model (MSE+multitask) drops to $82.1 \%$. For many error cases, our model predicts a close-to-correct segmentation, e.g., \#youknowyoumpttooearly, \#iseelondoniseefrance, which is also reflected by 


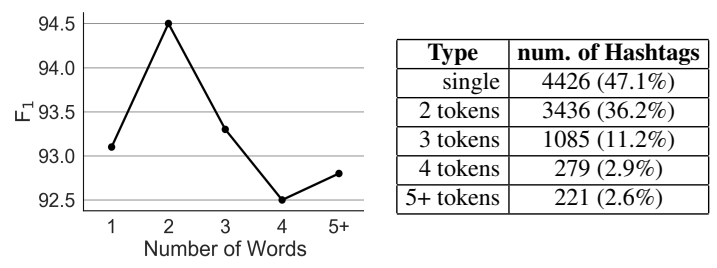

Figure 2: Token-level $\mathrm{F}_{1}$ scores (MSE+multitask) on hashtags of different lengths in the $\mathrm{STAN}_{\text {large }}$ test set.

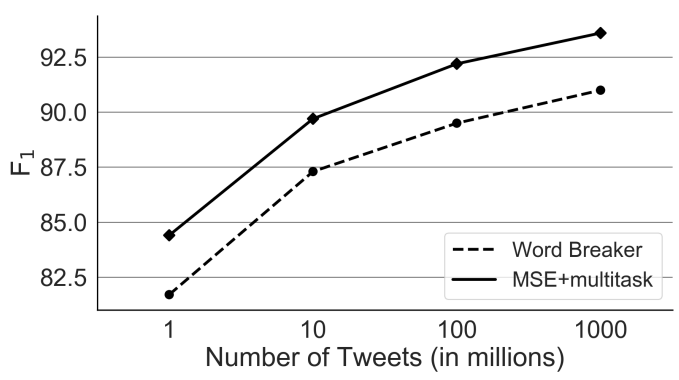

Figure 3: Token-level $F_{1}$ scores of our pairwise ranker (MSE+multitask) and Word Breaker on the STAN large $_{\text {. }}$ test set, using language models trained with varying amounts of data.

the higher token-level $F_{1}$ scores across hashtags with different lengths (Figure 2).

Size of the Language Model. Since our approach heavily relies on building a Twitter language model, we experimented with its sizes and show the results in Figure 3. Our approach can perform well even with access to a smaller amount of tweets. The drop in $\mathrm{F}_{1}$ score for our pairwise neural ranker is only $1.4 \%$ and $3.9 \%$ when using the language models trained on $10 \%$ and $1 \%$ of the total 1.1 billion tweets, respectively.

Time Sensitivity. Language use in Twitter changes with time (Eisenstein, 2013). Our pairwise ranker uses language models trained on the tweets from the year 2010. We tested our approach on a set of 500 random English hashtags posted in tweets from the year 2019 and show the results in Table 8. With a segmentation-level accuracy of $94.6 \%$ and average token-level $\mathrm{F}_{1}$ score of $95.6 \%$, our approach performs favorably on 2019 hashtags.

\begin{tabular}{llll}
\hline & A@1 & F 1 @ 1 & MRR \\
\hline Word Breaker w/ Twitter LM & 92.1 & 93.9 & 94.7 \\
Pairwise neural ranker (MSE+multitask) & $\mathbf{9 4 . 6}$ & $\mathbf{9 5 . 6}$ & $\mathbf{9 6 . 7}$ \\
\hline
\end{tabular}

Table 8: Evaluation results on 500 random hashtags from the year 2019 .

\section{Extrinsic Evaluation: Twitter Sentiment Analysis}

We attempt to demonstrate the effectiveness of our hashtag segmentation system by studying its impact on the task of sentiment analysis in Twitter (Pang et al., 2002; Nakov et al., 2016; Rosenthal et al., 2017). We use our best model (MSE+multitask), under the name HashtagMaster, in the following experiments.

\subsection{Experimental Setup}

We compare the performance of the BiLSTM+Lex (Teng et al., 2016) sentiment analysis model under three configurations: (a) tweets with hashtags removed, (b) tweets with hashtags as single tokens excluding the \# symbol, and (c) tweets with hashtags as segmented by our system, HashtagMaster. BiLSTM+Lex is a state-of-the-art open source system for predicting tweet-level sentiment (Tay et al., 2018). It learns a context-sensitive sentiment intensity score by leveraging a Twitterbased sentiment lexicon (Tang et al., 2014). We use the same settings as described by Teng et al. (2016) to train the model.

We use the dataset from the Sentiment Analysis in Twitter shared task (subtask A) at SemEval 2017 (Rosenthal et al., 2017). ${ }^{9}$ Given a tweet, the goal is to predict whether it expresses POSITIVE, NEGATIVE or NEUTRAL sentiment. The training and development sets consist of 49,669 tweets and we use 40,000 for training and the rest for development. There are a total of 4,840 tweets containing 12,128 hashtags in the SemEval 2017 test set, and our hashtag segmenter ended up splitting 6,975 of those hashtags present in 3,384 tweets.

\subsection{Results and Analysis}

In Table 9, we report the results based on the 3,384 tweets where HashtagMaster predicted a split, as for the rest of tweets in the test set, the hashtag segmenter would neither improve nor worsen the sentiment prediction. Our hashtag segmenter successfully improved the sentiment analysis performance by $2 \%$ on average recall and $\mathrm{F}_{1}^{P N}$ comparing to having hashtags unsegmented. This improvement is seemingly small but decidedly important for tweets where sentiment-related information is embedded in multi-word hashtags

\footnotetext{
${ }^{9}$ We did not use the Stanford Sentiment Analysis Dataset (Go and Huang, 2009), which was used to construct the STAN $_{\text {small }}$ and STAN $_{\text {large }}$ hashtag datasets, because of its noisy sentiment labels obtained using distant supervision.
} 


\begin{tabular}{lccc}
\hline & AvgR & $\mathbf{F}_{1}^{P N}$ & Acc \\
\hline Original tweets & $\mathbf{6 1 . 7}$ & 60.0 & 58.7 \\
- No Hashtags & $\mathbf{6 0 . 2}$ & 58.8 & 54.2 \\
+ Single-word & $\mathbf{6 2 . 3}$ & 60.3 & 58.6 \\
+ HashtagMaster & $\mathbf{6 4 . 3}$ & 62.4 & 58.6 \\
\hline
\end{tabular}

Table 9: Sentiment analysis evaluation on the 3384 tweets from SemEval 2017 test set using the BiLSTM+Lex method (Tang et al., 2014). Average recall (AvgR) is the official metric of the SemEval task and is more reliable than accuracy (Acc). $\mathrm{F}_{1}^{P N}$ is the average $F_{1}$ of positive and negative classes. Having the hashtags segmented by our system HashtagMaster (i.e., MSE+multitask) significantly improves the sentiment prediction than not ( $p<0.05$ for AvgR and $\mathrm{F}_{1}^{P N}$ against the single-word setup).

and sentiment prediction would be incorrect based only on the text (see Table 10 for examples). In fact, 2,605 out of the 3,384 tweets have multiword hashtags that contain words in the Twitterbased sentiment lexicon (Tang et al., 2014) and 125 tweets contain sentiment words only in the hashtags but not in the rest of the tweet.

\section{Other Related Work}

Automatic hashtag segmentation can improve the performance of many applications besides sentiment analysis, such as text classification (Billal et al., 2016), named entity linking (Bansal et al., 2015) and modeling user interests for recommendations (Chen et al., 2016). It can also help in collecting data of higher volume and quality by providing a more nuanced interpretation of its content, as shown for emotion analysis (Qadir and Riloff, 2014), sarcasm and irony detection (Maynard and Greenwood, 2014; Huang et al., 2018). Better semantic analysis of hashtags can also potentially be applied to hashtag annotation (Wang et al., 2019), to improve distant supervision labels in training classifiers for tasks such as sarcasm (Bamman and Smith, 2015), sentiment (Mohammad et al., 2013), emotions (Abdul-Mageed and Ungar, 2017); and, more generally, as labels for pre-training representations of words (Weston et al., 2014), sentences (Dhingra et al., 2016), and images (Mahajan et al., 2018).

\section{Conclusion}

We proposed a new pairwise neural ranking model for hashtag segmention and showed significant performance improvements over the state-of-theart. We also constructed a larger and more curated dataset for analyzing and benchmarking

\begin{tabular}{l}
\hline Ofcourse \#clownshoes \#altright \#IllinoisNazis \\
\hline \#FinallyAtpeaceWith people calling me "Kim \\
Fatty the Third" \\
\hline Leslie Odom Jr. sang that. \#ThankYouObama \\
\hline After some 4 months of vegetarianism .. it's all the \\
same industry. \#cutoutthecrap
\end{tabular}

Table 10: Sentiment analysis examples where our HashtagMaster segmentation tool helped. Red and blue words are negative and positive entries in the Twitter sentiment lexicon (Tang et al., 2014), respectively.

hashtag segmentation methods. We demonstrated that hashtag segmentation helps with downstream tasks such as sentiment analysis. Although we focused on English hashtags, our pairwise ranking approach is language-independent and we intend to extend our toolkit to languages other than English as future work.

\section{Acknowledgments}

We thank Ohio Supercomputer Center (Center, 2012) for computing resources and the NVIDIA for providing GPU hardware. We thank Alan Ritter, Quanze Chen, Wang Ling, Pravar Mahajan, and Dushyanta Dhyani for valuable discussions. We also thank the annotators: Sarah Flanagan, Kaushik Mani, and Aswathnarayan Radhakrishnan. This material is based in part on research sponsored by the NSF under grants IIS-1822754 and IIS-1755898, DARPA through the ARO under agreement number W911NF-17-C-0095, through a Figure-Eight (CrowdFlower) AI for Everyone Award and a Criteo Faculty Research Award to Wei $\mathrm{Xu}$. The views and conclusions contained in this publication are those of the authors and should not be interpreted as representing official policies or endorsements of the U.S. Government.

\section{References}

Muhammad Abdul-Mageed and Lyle Ungar. 2017. Emonet: Fine-grained emotion detection with gated recurrent neural networks. In Proceedings of the 55th Annual Meeting of the Association for Computational Linguistics, ACL, pages 718-728.

David Bamman and Noah A Smith. 2015. Contextualized Sarcasm Detection on Twitter. In Ninth International AAAI Conference on Web and Social Media, ICWSM, pages 574-577.

Piyush Bansal, Romil Bansal, and Vasudeva Varma. 2015. Towards Deep Semantic Analysis of Hashtags. In Proceedings of the 37th European Conference on Information Retrieval, ECIR, pages 453-464. 
Giacomo Berardi, Andrea Esuli, Diego Marcheggiani, and Fabrizio Sebastiani. 2011. ISTI@TREC Microblog Track 2011: Exploring the Use of Hashtag Segmentation and Text Quality Ranking. In Text REtrieval Conference (TREC).

Taylor Berg-Kirkpatrick, David Burkett, and Dan Klein. 2012. An Empirical Investigation of Statistical Significance in NLP. In Proceedings of the 2012 Joint Conference on Empirical Methods in Natural Language Processing and Computational Natural Language Learning, EMNLP-CoNLL, pages 9951005.

Belainine Billal, Alexsandro Fonseca, and Fatiha Sadat. 2016. Named Entity Recognition and Hashtag Decomposition to Improve the Classification of Tweets. In Proceedings of the 2nd Workshop on Noisy User-generated Text (WNUT), COLING, pages 102-111.

Thorsten Brants and Alex Franz. 2006. Web 1T 5-gram Version 1. Linguistic Data Consortium (LDC).

Arda Çelebi and Arzucan Özgür. 2016. Segmenting Hashtags using Automatically Created Training Data. In Proceedings of the Tenth International Conference on Language Resources and Evaluation, LREC, pages 2981-2985.

Arda Çelebi and Arzucan Özgür. 2017. Segmenting Hashtags and Analyzing Their Grammatical Structure. Journal of Association For Information Science and Technology (JASIST), 69(5):675-686.

Ohio Supercomputer Center. 2012. Oakley supercomputer. http://osc.edu/ark:/19495/ hpc0cvon.

Stanley F Chen and Joshua Goodman. 1999. An empirical study of smoothing techniques for language modeling. Computer Speech \& Language, 13(4):359-394.

Tao Chen, Xiangnan He, and Min-Yen Kan. 2016. Context-aware Image Tweet Modelling and Recommendation. In Proceedings of the 24th ACM International Conference on Multimedia, MM, pages 1018 1027.

Dan Ciresan, Ueli Meier, and Jürgen Schmidhuber. 2012. Multi-column Deep Neural Networks for Image Classification. In Proceedings of the 2012 IEEE Conference on Computer Vision and Pattern Recognition, CVPR, pages 3642-3649.

William W Cohen, Robert E Schapire, and Yoram Singer. 1998. Learning to Order Things. In Advances in Neural Information Processing Systems, NIPS, pages 451-457.

Thierry Declerck and Piroska Lendvai. 2015. Processing and normalizing hashtags. In Proceedings of the International Conference Recent Advances in Natural Language Processing, RANLP, pages 104-109.
Bhuwan Dhingra, Zhong Zhou, Dylan Fitzpatrick, Michael Muehl, and William Cohen. 2016. Tweet2Vec: Character-Based Distributed Representations for Social Media. In Proceedings of the 54th Annual Meeting of the Association for Computational Linguistics, ACL, pages 269-274.

Jacob Eisenstein. 2013. What to do about bad language on the Internet. In Proceedings of the 2013 Conference of the North American Chapter of the Association for Computational Linguistics, NAACL, pages 359-369.

Tim Finin, Will Murnane, Anand Karandikar, Nicholas Keller, Justin Martineau, and Mark Dredze. 2010. Annotating named entities in Twitter data with crowdsourcing. In Proceedings of the Workshop on Creating Speech and Language Data with Amazon's Mechanical Turk, NAACL, pages 80-88.

Bhayani R. Go, A. and L. Huang. 2009. Twitter Sentiment Classification using Distant Supervision. CS224N Project Report, Stanford.

Irving J Good. 1953. The population frequencies of species and the estimation of population parameters. Biometrika, 40(3-4):237-264.

David Graff and Christopher Cieri. 2003. English Gigaword LDC2003T05. Linguistic Data Consortium $(L D C)$.

Bo Han and Timothy Baldwin. 2011. Lexical Normalisation of Short Text Messages: Makn Sens a\# twitter. In Proceedings of the 49th Annual Meeting of the Association for Computational Linguistics, ACL, pages $368-378$.

Kenneth Heafield. 2011. KenLM: Faster and Smaller Language Model Queries. In Proceedings of the Sixth Workshop on Statistical Machine Translation, WMT, pages 187-197.

Kenneth Heafield. 2013. Efficient Language Modeling Algorithms with Applications to Statistical Machine Translation. Ph.D. thesis, Carnegie Mellon University.

Mark Hopkins and Jonathan May. 2011. Tuning as ranking. In Proceedings of the Conference on Empirical Methods in Natural Language Processing, EMNLP.

Hen-Hsen Huang, Chiao-Chen Chen, and Hsin-Hsi Chen. 2018. Disambiguating false-alarm hashtag usages in tweets for irony detection. In Proceedings of the 56th Annual Meeting of the Association for Computational Linguistics, ACL, pages 771-777.

Diederik P. Kingma and Jimmy Ba. 2014. Adam: A Method for Stochastic Optimization. In Proceedings of the 3rd International Conference for Learning Representations, ICLR. 
Reinhard Kneser and Hermann Ney. 1995. Improved backing-off for $\mathrm{m}$-gram language modeling. In Proceedings of the 1995 International Conference on Acoustics, Speech, and Signal Processing, ICASSP, pages 181-184.

Philipp Koehn and Kevin Knight. 2003. Empirical methods for compound splitting. In Proceedings of the tenth conference on European chapter of the Association for Computational Linguistics, EACL, pages 187-194.

Dhruv Mahajan, Ross Girshick, Vignesh Ramanathan, Kaiming He, Manohar Paluri, Yixuan Li, Ashwin Bharambe, and Laurens van der Maaten. 2018. Exploring the Limits of Weakly Supervised Pretraining. In Tech Report.

Diana Maynard and Mark A Greenwood. 2014. Who cares about sarcastic tweets? Investigating the impact of sarcasm on sentiment analysis. In Proceedings of the 9th International Conference on Language Resources and Evaluation, LREC, pages 4238-4243.

Saif Mohammad, Svetlana Kiritchenko, and Xiaodan Zhu. 2013. NRC-Canada: Building the state-of-theart in sentiment analysis of tweets. In Proceedings of the Seventh International Workshop on Semantic Evaluation, SemEval, pages 321-327.

Preslav Nakov, Sara Rosenthal, Svetlana Kiritchenko, Saif M. Mohammad, Zornitsa Kozareva, Alan Ritter, Veselin Stoyanov, and Xiaodan Zhu. 2016. Developing a successful SemEval task in sentiment analysis of Twitter and other social media texts. Language Resources and Evaluation, 50(1):35-65.

Dong Nguyen, Barbara McGillivray, and Taha Yasseri. 2018. Emo, love and god: making sense of urban dictionary, a crowd-sourced online dictionary. Royal Society Open Science, 5(5):172320.

Ozer Ozdikis, Pinar Senkul, and Halit Oguztuzun. 2012. Semantic Expansion of Hashtags for Enhanced Event Detection in Twitter. In Proceedings of the 1st international Workshop on Online Social Systems.

Bo Pang, Lillian Lee, and Shivakumar Vaithyanathan. 2002. Thumbs up? Sentiment Classification using Machine Learning Techniques. In Proceedings of the Conference on Empirical Methods in Natural Language Processing, EMNLP, pages 79-86.

M. Parakhin and P. Haluptzok. 2009. Finding the Most Probable Rranking of Objects with Probabilistic Pairwise Preferences. In Proceedings of the 10th International Conference on Document Analysis and Recognition, ICDAR, pages 616-620.

Fuchun Peng and Dale Schuurmans. 2001. A hierarchical em approach to word segmentation. In NLPRS, pages 475-480.
Ashequl Qadir and Ellen Riloff. 2014. Learning emotion indicators from tweets: Hashtags, hashtag patterns, and phrases. In Proceedings of the 2014 Conference on Empirical Methods in Natural Language Processing, EMNLP, pages 1203-1209.

Jack Reuter, Jhonata Pereira-Martins, and Jugal Kalita. 2016. Segmenting twitter hashtags. International Journal on Natural Language Computing, 5:23-36.

Alan Ritter, Sam Clark, Oren Etzioni, et al. 2011. Named Entity Recognition in Tweets: An Experimental Study. In Proceedings of the Conference on Empirical Methods in Natural Language Processing, EMNLP, pages 1524-1534.

Sara Rosenthal, Noura Farra, and Preslav Nakov. 2017. SemEval-2017 task 4: Sentiment Analysis in Twitter. In Proceedings of the 11th International Workshop on Semantic Evaluation, SemEval, pages 502-518.

Justin Sampson, Fred Morstatter, Liang Wu, and Huan Liu. 2016. Leveraging the implicit structure within social media for emergent rumor detection. In Proceedings of the 25th ACM International on Conference on Information and Knowledge Management, CIKM, pages 2377-2382.

C. Simeon, H. J. Hamilton, and R. J. Hilderman. 2016. Word segmentation algorithms with lexical resources for hashtag classification. In Proceedings of the 2016 IEEE International Conference on Data Science and Advanced Analytics (DSAA), pages 743-751.

Richard Sproat and Chilin Shih. 1990. A statistical method for finding word boundaries in chinese text. Computer Processing of Chinese and Oriental Languages, 4(4):336-351.

Andreas Stolcke. 2002. SRILM - An Extensible Language Modeling Toolkit. In Proceedings of the 7th International Conference on Spoken Language Processing, ICSLP, pages 901-904.

Duyu Tang, Furu Wei, Bing Qin, Ming Zhou, and Ting Liu. 2014. Building Large-Scale Twitter-Specific Sentiment Lexicon : A Representation Learning Approach. In Proceedings of the 25th International Conference on Computational Linguistics, COLING, pages 172-182.

Yi Tay, Anh Tuan Luu, Siu Cheung Hui, and Jian Su. 2018. Attentive gated lexicon reader with contrastive contextual co-attention for sentiment classification. In Proceedings of the 2018 Conference on Empirical Methods in Natural Language Processing, EMNLP, pages 3443-3453.

Zhiyang Teng, Duy Tin Vo, and Yue Zhang. 2016. Context-Sensitive Lexicon Features for Neural Sentiment Analysis. In Proceedings of the 2016 Conference on Empirical Methods in Natural Language Processing, EMNLP, pages 1629-1638. 
Kuansan Wang, Christopher Thrasher, and BoJune Paul Hsu. 2011. Web Scale NLP: A Case Study on URL Word Breaking. In Proceedings of the 20th International Conference on World Wide Web, WWW, pages 357-366.

Yue Wang, Jing Li, Irwin King, Michael R. Lyu, and Shuming Shi. 2019. Microblog Hashtag Generation via Encoding Conversation Contexts. In Proceedings of the North American Chapter of the Association for Computational Linguistics (NAACL).

Jason Weston, Sumit Chopra, and Keith Adams. 2014. \# tagspace: Semantic embeddings from hashtags. In Proceedings of the 2014 Conference on Empirical Methods in Natural Language Processing, EMNLP, pages 1822-1827.

Nianwen Xue and Libin Shen. 2003. Chinese word segmentation as LMR tagging. In Proceedings of the second SIGHAN workshop on Chinese Language Processing, SIGHAN, pages 176-179.

\section{A Appendix}

\section{A.1 Word-shape rules}

Our model uses the following word shape rules as boolean features. If the candidate segmentation $s$ and its corresponding hashtag $h$ satisfies a word shape rule, then the boolean feature is set to True.

\begin{tabular}{r|l}
\hline Rule & Hashtag $\rightarrow$ Segmentation \\
\hline Camel Case & $\mathrm{XxxXxx} \rightarrow \mathrm{Xxx}+\mathrm{Xxx}$ \\
Consonants & $\operatorname{cccc} \rightarrow \mathrm{cccc}$ \\
Digits as prefix & ddwwww $\rightarrow$ dd + wwww \\
Digits as suffix & wwwwdd $\rightarrow$ wwww + dd \\
Underscore & www_www $\rightarrow$ www $+_{-}+$www \\
\hline
\end{tabular}

Table 11: Word-shape rule features used to identify good segmentations. Here, $X$ and $x$ represent capitalized and non-capitalized alphabetic characters respectively, $c$ denotes consonant, $d$ denotes number and $w$ denotes any alphabet or number. 